\title{
VALORACIÓN DE LAS COMPETENCIAS DE LOS DOCENTES EN EL CONTEXTO UNIVERSITARIO
}

\section{ASSESSMENT OF THE COMPETENCES OF TEACHERS IN THE UNIVERSITY CONTEXT}

\author{
MSc. Mary Ramona Bohórquez Casadiegos ${ }^{a}$, MSc. Claudia Marcela Durán Chinchilla ${ }^{\text {b }}$, MSc. \\ Alveiro Alonso Rosado Gómez ${ }^{\mathrm{c}}$ \\ ${ }^{a}$ Universidad Francisco de Paula Santander, Grupo de Investigación de la Facultad de \\ Educación, Artes y Humanidades (GIFEAH) \\ Calle 11 No. 23 - 17, Ocaña, Colombia, mrbohorquezc@ufpso.edu.co \\ ${ }^{\mathrm{b}}$ Universidad Francisco de Paula Santander, Grupo de Investigación de la Facultad de \\ Educación, Artes y Humanidades (GIFEAH) \\ Casa 50 Jardín de la Rosa, Ocaña, Colombia, cmduranc@ufpso.edu.co \\ ${ }^{\mathrm{c}}$ Universidad Francisco de Paula Santander, Grupo de Investigación en Desarrollo Tecnológico \\ en Ingeniería (GITYD) \\ Cra 26 No. 4-41 Marabel, Ocaña, Colombia, aarosadog@ufpso.edu.co
}

Fecha de recepción: 01-05-2017

Fecha de aprobación: 22-06-2017

Resumen: El presente trabajo de investigación tiene como objetivo, valorar las competencias de los docentes de la Universidad Francisco de Paula Santander Ocaña (UFPSO), buscando organizar el desempeño de su personal docente y la productividad de los programa.

La investigación fue de tipo descriptivo exploratorio, se planteó mediante un diseño cualitativo colaborativo, tipo survey, mediante cuestionario. La población de la cual se obtuvo la información, estuvo conformada por 352 docentes. Se utilizó la técnica documental y se recurrió al cuestionario. Para la identificación de las competencias se utilizó el método de expertos y para la priorización de las mismas el Delphy.

Finalmente, se determinaron las competencias de área y las competencias de rol, con los datos obtenidos se realizó la escala de valoración, la cual permitiera evaluar el despeño docente.

Palabras clave: Competencia, docencia, competencia genérica y competencia transversal, desempeño docente. 
Abstract: The present work of investigation aims, the design of a competency of the Francisco de Paula Santander University Ocaña, looking for organizing the performance of its teacher's staff and productivity of the program.

The research was descriptive exploratory type, a qualitative collaborative design, type survey, through questionnaire. The population from which Information was obtained, was confirmed by 352 teacher. A documental technique was used and was resorted with the questionnaire. For identifying the competencies, an Expert method was used and for the prioritization of them, the Delphi method.

Finally, area and role-plays competencies were determined; with the data obtained a rating scale was performed, which will evaluate the teacher's performance.

Keywords: Competition- Teaching- generic competition and cross competition- teacher's performance.

\section{INTRODUCCIÓN}

En el medio cambiante y en la era de globalización, muchas organizaciones centralizan sus metas y atención en grandes inversiones tecnológicas como canal principal de innovación y crecimiento, otorgando menor peso al talento humano que poseen.

El termino competencia nace del auge de los modelos de desarrollo de la globalización cultural y la apertura económica, las competencias toman fuerza en las políticas educativas, máxime para entrar a definir lo que se debe esperar de la formación del talento humano ya que es a quien le corresponde asistir a los nuevos tipos: de organizaciones, trabajo, e inserción laboral (Ruiz Iglesias, 2005).

Las prácticas de la evaluación de desempeño, no son recientes, desde que una persona empleó a otra el trabajo de ésta, fue evaluado. En varios escenarios y antes de la segunda guerra mundial se han desarrollado sistemas de evaluación, sin embargo, posterior a esta guerra fue cuando se implementó la evaluación de desempeño en las organizaciones (Chiavenato, 2001).
En cuanto a lo que corresponde al término competencia es definido como un conocimiento determinado que pone en juego capacidades en un campo disciplinar determinado o como la habilidad para resolver tareas o problemas concretos en distintas situaciones (Bogoya \& Torrado, 2000).

Se afirma que algunas experiencias han contribuido notablemente a la expansión del enfoque basado en competencias: una de ellas es el Proyecto Tuning en Europa y América Latina, cuyo objetivo ha sido la generación de espacios que permitan acordar las estructuras educativas de los diferentes países para su mutua comprensión, comparación y reconocimiento. Todo ello bajo el supuesto de que en una etapa de franca internacionalización, la universidad como actor social, enfrentará retos y responsabilidades, al margen del lugar geográfico, en donde se encuentre (Rueda Beltrán, 2009).

En ese sentido, el proyecto Tuning, define la competencia como una mezcla de particularidades, en relación a conocimientos, destrezas, cualidades y responsabilidades, que representan los 
resultados finales de del aprendizaje, (Bezanilla, 2003). Dentro del proyecto se proponen las competencias específicas son las de naturaleza técnica, las cuales proceden de las exigencias de un contexto; en tal sentido, aportan identidad y firmeza social y profesional al perfil formativo, de tal forma que se pueden dividir según su ámbitos (Pavié Nova, 2012). En cuanto a las competencias genéricas son, la descripción del rol o roles esenciales identificados en la figura profesional, en forma de objetivos de producción o de formación, se relacionan con los comportamientos y actitudes comunes, en las labores de diferentes ámbitos de producción, por ejemplo: capacidad para trabajar en equipo, saber planificar, habilidad para negociar, etc. (Domínguez, 2004).

En ese sentido, la diferenciación entre competencias genéricas y específicas, está dada en la medida que las primeras son susceptibles de aplicarse en situaciones variadas, permitiendo pasar de un contexto a otro; en tanto que las segundas, serían de utilidad más restringida (Tejada \& Navio, 2005).

En el ámbito educativo, desde el pragmatismo epistemológico, se propone pensar en las competencias como actuaciones que han de identificar aquello que necesita cualquier persona para dar respuesta a los problemas que se enfrentará a lo largo de su vida (Zabala \& Laia, 2007).

En lo que respecta a la declaración de que quien sabe "ya sabe hacer y sabe ser", lleva a suponer que los procedimientos, habilidades, actitudes y valores no son objetos de la educación, y por lo tanto, no son ni deberían ser contenidos de la enseñanza. Para estos autores se ha dejado de lado que las acciones resultan de una integración de lo conceptual (saber), lo procedimental (saber hacer) y lo actitudinal (saber ser) (Valladares, 2010).

De la misma manera, se considera que una docencia por competencias requiere un docente capaz de trabajar con estrategias y dispositivos de formación fundados en un modelo pedagógico que considere momentos de planeación auténtica, en la cual el profesor diseña situaciones-problema cercanas a la realidad del estudiante y orientadas a que éste movilice sus recursos cognitivos para encontrar soluciones a las situaciones planteadas y, en ese proceso, tome decisiones y autorregule su aprendizaje (Guzmán Ibarra \& Marín Uribe, 2015).

Para otros estudiosos, las competencias se sintetizan en tres elementos: la información, desarrollo de una habilidad y valoración o actitud (Díaz Barriga, 2006); Sin embargo también son consideradas como la integración de distintos saberes: saber ser, saber hacer, saber conocer y saber convivir (Tobón, 2007)

Otro concepto importante para mencionar, en cuanto a competencia, es en el cual se definen la misma como una capacidades de desempeño: saber hacer, el ser y el convivir, en tal sentido, dice el autor que está relacionada con habilidades, actitudes que el individuo posee a la hora de solucionar un problema (Arbeláez, Corredor, \& Pérez, 2009).

En el ámbito de la docencia universitaria, el concepto de competencia docente puede ser entendido de manera amplia como la manera de desempeñarse en la interacción social, en los distintos contextos y situaciones diarias que implican la práctica social de la profesión (Guzmán Ibarra \& Marín Uribe, 2011); Se plantea también que la competencia docente está conexa con las prácticas educativas y su incidencia en el 
desempeño profesional (Nieto \& Díaz, 2009).

Las competencias docentes se expresan pues en desempeños genéricos que corresponden a las características de las funciones y acciones propias de prácticas docentes específicas, del quehacer cotidiano del docente que, para efectos prácticos de entendimiento, corresponden a la organización y ejercicio del trabajo escolar que el docente, de manera general, realiza en tres distintos momentos: antes (planeación macro y micro), durante (en el aula) y después, (en el contexto), (García Cabrero , Loredo, Luna, \& Rueda, 2008).

La competencia docente se debe focalizar más en el aprendizaje que en la enseñanza y por su puesto el rol del estudiante en cuanto a la construcción del conocimiento, esto lleva a que el docente se centre más en cómo facilitar el aprendizaje, es decir partir del qué, el cómo y para qué aprender (Cano García, 2008), de tal forma, el docente de hoy debe desarrollar habilidades, actitudes y valores que generen a su vez generen competencias en sus estudiantes (Castro, 2007).

Así mismo, las funciones docentes se pueden clasificar a través de cuatro funciones: didácticas, tutoriales, vinculación con el medio social, formación e Innovación (Sarramorra, 2005). En cuanto a la didáctica se requiere que el docente conozca la disciplina que enseña y con base en ello desarrolle una función didáctica de la misma; en ese sentido, la didáctica está relacionada con lo que el docente cree que el estudiante debe aprender y cómo lo aprende (Putman \& Borko, 2000).

Con relación a la función tutorial muestra que enseñar no es solo explicar unos contenidos, sino dirigir globalmente el procesos formativo de los alumnos, de la misma manera plantea que es la vinculación con el medio social, como la integración del docente con la organización y disposición para trabajar colaborativamente con los demás docentes por lo que se hace indispensable que el docente trabaje en equipo, y se comprometa con la institución (Zabalza M., 2003).

En ese sentido, se sugieren las siguientes competencias desde y para los profesionales: competencias técnicas (saber), competencias metodológicas (saber hacer), competencias participativas (saber estar), competencias personales (saber ser) (Echeverría, 2002), esto lleva a concluir que los docentes deben estar en constante formación lo cual le permita estar en tono a las exigencias culturales, sociales, políticas y de globalización que el mundo actual exige en cada uno de los profesionales (Pérez Gómez, 2007).

Hay también, tres dimensiones importantes en cuanto a las competencias docentes de las cuales se pueden mencionar: Dimensión del profesor el cual permite determinar qué se espera que haga el profesional; la dimensión personal, relacionado con fuentes de satisfacción o insatisfacción en el trabajo y la dimensión laboral relacionados con incentivos y condiciones laborales propiamente dichas (Zabalza M., 2002). Por tanto, para que se asegure una docencia con calidad se requiere que se establezca un perfil docente flexible y capaz a de adaptarse a los continuos cambios de la sociedad (Bozu \& Canto, 2009).

Teniendo en cuanta las anteriores apreciaciones, el docente no debe centrarse únicamente en la transmisión de contenidos, sino más bien debe dedicarse a la gestión del aprendizaje de los estudiantes, orientando, guiando y ofreciendo las pautas y medios necesarios para que él mismo, de la manera más autónoma posible, para que sea el 
constructor de su propio proceso de aprendizaje (Zabalza M., 2009). De tal manera que el docente debe dominar tanto un perfil científico-metodológico, referente a las disciplinas de las que es responsable, como un perfil didáctico, ocupando las competencias que le exige la tarea docente: planificar, ejecutar y evaluar, de tal forma que el profesor debe ser conocedor de la disciplina que imparte y especialista en el diseño, desarrollo, análisis y evaluación de su aplicación (Barraza, 2007).

Desde esa perspectiva, el estudio abordó y definió lo correspondiente a la competencias transversales: de área y de rol; y por supuesto las competencias genéricas.

\section{METODOLOGÍA}

En la tabla 1, resume los aspectos metodológicos que fueron tenidos en cuenta para la realización de la investigación.

Tabla 1. Diseño Metodológico.

\begin{tabular}{|c|c|}
\hline Elemento & Descripción \\
\hline $\begin{array}{l}\text { Tipo de } \\
\text { investigación }\end{array}$ & Descriptiva \\
\hline $\begin{array}{l}\text { Método de } \\
\text { investigación } \\
\text { Fuentes }\end{array}$ & $\begin{array}{l}\text { Deductivo, teniendo en cuenta que se } \\
\text { parte de lo general a lo particular. } \\
\text { Primarias y secundarias }\end{array}$ \\
\hline $\begin{array}{l}\text { Técnicas de } \\
\text { recolección }\end{array}$ & Censal \\
\hline Instrumentos & $\begin{array}{l}\text { Cuestionarios, estructurados o panel de } \\
\text { expertos. }\end{array}$ \\
\hline $\begin{array}{l}\text { Modo } \\
\text { aplicación }\end{array}$ & Directa \\
\hline $\begin{array}{l}\text { Definición } \\
\text { población }\end{array}$ & $\begin{array}{l}\text { Docentes catedráticos, ocasionales y de } \\
\text { planta tiempo completo. }\end{array}$ \\
\hline $\begin{array}{l}\text { Proceso } \\
\text { muestreo }\end{array}$ & $\begin{array}{l}\text { Para que todos los docentes de los } \\
\text { distintos programas académicos } \\
\text { tuvieran la posibilidad de participar en } \\
\text { el estudio se utilizó el muestreo } \\
\text { probabilístico sistemático. }\end{array}$ \\
\hline $\begin{array}{l}\text { Tiempo } \\
\text { aplicación }\end{array}$ & 1 mes. \\
\hline
\end{tabular}

Como se expresa en la tabla anterior, la población seleccionada para el estudio fueron los docentes de la UFPSO, los cuales suman 352 docentes, adscritos a los distintos departamentos académicos; en tal sentido, y para que cada departamento tuviera representación y para que hubiese mayor validez y confiabilidad, se tomó una muestra representativa, aplicando la formula estadística, en la que el nivel de confianza fue del 95\%, margen de error del $5 \%$ dando como resultado: 38 docentes, luego se tomó el factor de selección para que cada departamento tuviera la probabilidad de participar en el estudio, como se muestra en la tabla 2.

Tabla 2. Submuestra.

\begin{tabular}{lcc}
\hline \multicolumn{1}{c}{$\begin{array}{c}\text { Departamento } \\
\text { Académico }\end{array}$} & $\begin{array}{c}\text { Número de } \\
\text { docentes }\end{array}$ & Submuestra \\
\hline $\begin{array}{l}\text { Humanidades } \\
\text { Ciencias Básicas }\end{array}$ & 103 & 11 \\
$\begin{array}{l}\text { Ciencias administrativa y } \\
\text { contable }\end{array}$ & 43 & 5 \\
$\begin{array}{l}\text { Ingenieras } \\
\text { Ciencias Agrarias y del }\end{array}$ & 102 & 9 \\
$\begin{array}{l}\text { ambiente } \\
\text { Total }\end{array}$ & 22 & 10 \\
\hline
\end{tabular}

Fuente: Elaboración propia.

Obtenida la submuestra, se acudió a sujetos voluntarios, para aplicar el instrumento, en este caso, se acudió a la encuesta, la cual estuvo conformada por quince preguntas cerradas con opción múltiple, una vez aplicado el instrumento y obtenidos los resultados se realizó un panel de expertos.

\section{RESULTADOS}

Los resultados se sintetizan en tres productos: Identificación de competencias, comportamientos asociados y escala de valoración. En lo que respecta a las competencias transversales, como se muestra en la tabla 3, el estudio propone quince competencias que debe poseer un docente.

Tabla 3. Competencias transversales.

\begin{aligned} \hline Numero & \multicolumn{1}{c}{ Descripción } \\ \hline 1 & Trabajo en equipo \\ 2 & Compromiso ético \end{aligned}


3 Resolución de problemas y conflictos

Habilidad de producción y comprensión de textos

Formulación, Dirección y Gestión de proyectos

6 Manejo de las Tics

7 Capacidad Investigativa

Capacidad de Actualización del conocimiento

9 Adaptabilidad al cambio

10 Respeto por las normas, principios y valores

10 Institucionales

11 Productividad

12 Liderazgo

13 Conocimiento del entorno de su profesión

14 Capacidad para la toma de decisiones

15 Capacidad para la formación de estudiante

Fuente: Elaboración propia.

Para la priorización de las competencias (transversales, genéricas o de área y de rol), se utilizó método Delphi por su capacidad de predicción con base en la apreciación sistemática de un juicio intuitivo emitido por un grupo de expertos; es decir, se procede por medio de la interrogación a los elementos muéstrales con la ayuda de cuestionarios sucesivos, a fin de poner de manifiesto convergencias de opiniones y deducir eventuales consensos (Cruz \& Martínez, 2012). La encuesta se llevó a cabo de una manera anónima para evitar los efectos de "líderes". El objetivo de los cuestionarios sucesivos, fue el de "disminuir el espacio intercuartil, precisando la mediana".

A partir de la priorización se elabora el listado de las competencias de área y de rol que los docentes de la UFPSO deben tener.

Realizada la priorización de las competencias se procede a la aplicación de la media armónica, se elimina la competencia ubicada en la posición No 10 , cuyo promedio $(4,2)$ es menor del resto de competencias. Este valor, hace referencia a: capacidad crítica y autocrítica.
Tabla 4. Priorización de competencias de área.

\begin{tabular}{|c|c|c|c|c|c|c|c|c|c|c|c|}
\hline Competencias & 1 & 2 & 3 & 4 & 5 & 6 & 7 & 8 & 9 & 10 & 11 \\
\hline $\begin{array}{l}\text { Evaluación de } \\
\text { expertos } \\
\text { primera ronda. }\end{array}$ & 5 & 4,4 & 5 & 5 & 4,7 & 5 & 5 & 5 & 5 & 4,7 & 5 \\
\hline $\begin{array}{l}\text { Evaluación de } \\
\text { expertos } \\
\text { segunda } \\
\text { ronda. }\end{array}$ & 4,8 & 4,6 & 3,8 & 4 & 5 & 4,4 & 4,4 & 5 & 4,2 & 3,8 & 4,2 \\
\hline Promedio & 4,9 & 4,5 & 4,3 & 5 & 4,9 & 4,7 & 4,7 & 5 & 4,6 & 4,2 & 4,6 \\
\hline
\end{tabular}

Fuente: Elaboración propia.

Partiendo de esos datos se plantea en las tablas 5 y 6 , un cuadro de competencias de rol; a partir del mismo se determinan las competencias de área que debe tener el docente de la UFPSO.

Tabla 5. Competencias de rol.

\begin{tabular}{l} 
Ítem \\
\hline 1. Facilidad para la generación de acuerdo y \\
soluciones multilaterales duraderas. \\
2. Interacción con los diferentes agentes de la \\
comunidad educativa de manera precisa y oportuna. \\
3. Pensamiento Estratégico. \\
4. Intervención efectiva y oportuna ante situaciones \\
de conflicto generada en el entorno de trabajo. \\
5. Innovación del conocimiento. \\
6. Influencia positiva en el comportamiento de otros \\
alcanzando compromisos para el logro de metas \\
comunes. \\
7. Seguimiento a los compromisos adquiridos por las \\
partes. \\
8. Aprovechamiento de los conocimientos y \\
habilidades individuales para el potenciamiento \\
grupal. \\
9. Obtención y distribución de diferentes recursos en \\
forma estratégica en términos de personas, medios y \\
tiempo. \\
10. Conocimiento empresarial \\
uente: Elaboración propia.
\end{tabular}

Fuente: Elaboración propia.

Tabla 6. Competencias de área.

\begin{tabular}{l} 
Ítem \\
\hline 1. Facilidad para la generación de acuerdo y \\
soluciones multilaterales duraderas. \\
2. Interacción con los diferentes agentes de la \\
comunidad educativa de manera precisa y oportuna. \\
3. Pensamiento Estratégico. \\
4. Intervención efectiva y oportuna ante situaciones \\
de conflicto generada en el entorno de trabajo. \\
5. Innovación del conocimiento. \\
6. Influencia positiva en el comportamiento de otros \\
alcanzando compromisos para el logro de metas \\
comunes. \\
7. Seguimiento a los compromisos adquiridos por las \\
partes. \\
8. Aprovechamiento de los conocimientos y \\
habilidades individuales para el potenciamiento \\
grupal. \\
9. Obtención y distribución de diferentes recursos en \\
forma estratégica en términos de personas, medios y \\
tiempo. \\
10. Conocimiento empresarial \\
uente: Elaboración propia.
\end{tabular}




\section{DISCUSIÓN}

En la definición de las competencias anteriormente mencionadas, se tuvo en cuenta el concepto de Pavié, en el cual manifiesta que las competencias transversales, se pueden dividir también según los siguientes ámbitos: de acuerdo al conocimiento relacionadas con lo que un docente posee en cuanto a su disciplina y cómo transmite los conocimientos; los profesionales teniendo en cuenta la habilidad o habilidades comunicativas y de interacción con los estudiantes y los académicos lo relacionado con la habilidad para investigar y solucionar problemas vinculados con el aprendizaje de los estudiantes y por supuesto reflexionar sobre el mismo proceso académico, es decir auto reflexionarse (Pavié, 2011).

Una vez identificadas las competencias, se procede a definir las competencias halladas, describiendo el rol o roles esenciales identificados en la figura profesional en forma de objetivos de producción o de formación. Se relacionan con los comportamientos y actitudes comunes en las labores de diferentes ámbitos de producción (Domínguez, 2004).

\subsection{Definición de competencias}

Se elabora un concepto de la competencia, que muestre a que hace referencia dicha competencia; por ejemplo:

Nombre de la Competencia. Trabajo en equipo
Definición. Trabajar con otros de forma conjunta y de manera participativa, integrando esfuerzos para la consecución de las metas institucionales comunes.

\section{Comportamientos}

Asociados.

Determinadas las competencias se establecen unas posibles situaciones, relacionadas con el desempeño que van desde normal hasta destacados:

Coopera en distintas situaciones y comparte información.

Aporta sugerencias, ideas y opiniones.

Planifica las propias acciones teniendo en cuenta la repercusión de las-mismas para la consecución de los objetivos grupales.

Respeta criterios dispares y distintas opiniones del equipo.

Estos grados de conductas observables identificadas en el paso anterior, hacen relación literalmente con lo que se conoce como los indicadores de dominio que para el efecto son definidos con un conjunto de ítems o frases cortas referidas a conductas observables, que permiten evaluar el dominio de la competencia considerando, uno por uno, todo el conjunto de indicadores. En este caso, la brecha positiva o negativa resultará de la puntuación obtenida, considerando si el desempeño de la persona con respecto al indicador es A, B o $\mathrm{C}$, donde A podría significar "satisface plenamente o supera las exigencias del cargo", B "satisface las exigencias mínimas" y C "requiere entrenamiento formativo para alcanzar el desempeño requerido", con respecto al indicador. Para cada letra u otro 
signo escogido, habrá una puntuación, y el dominio se expresará en un puntaje total.

Escala de valoración. Los indicadores fueron definidos de acuerdo a las variables así: Análisis de competencias, se definieron cuatro indicadores los cuales coinciden con los grados (A, B, C y D) de los comportamientos asociados. Para esta dimensión el indicador va estar fundamentado en la existencia o no del producto de carácter científico, tecnológico o técnico. Trabajo pedagógico. Al igual que en el análisis de competencias, se definieron cuatro criterios: destacado, competente, básico e insatisfactorio.

Criterios de calificación de la evaluación. Los puntos a asignar fueron establecidos en un total de 100, los cuales abarcarían un $100 \%$ que se distribuirían así: $60 \%$ al análisis de las competencias, $10 \%$ a la productividad y $30 \%$ al trabajo pedagógico. Se establece un mayor porcentaje a al análisis de las competencias, teniendo que el punto de partida para la determinación del perfil docente apropiado para la facultad. El menor porcentaje dado a la productividad se fundamenta en el escaso desarrollo de ésta dimensión, la cual en la facultad y casi que en la Institución se encuentra en estado naciente.

A los indicadores tanto del análisis de competencias como de evaluación del trabajo pedagógico se le asignaron los siguientes puntajes: $\mathrm{A}=5$ puntos, $\mathrm{B}=4$ puntos, $\mathrm{C}=3$ puntos y $\mathrm{D}=2$ puntos.

Para la dimensión productividad, los 10 puntos asignados serían distribuidos entre las siete variables a medir, correspondiendo a cada una 1.43 puntos.

Conforme a los resultados obtenidos se otorgarán las categorías siguientes: Para las diferentes dimensiones:

Insatisfactorio: si el docente cumple con menos del $54 \%$ de los indicadores correspondiente y por tanto exige mejoramiento.

Básico: si el docente cumple con $69 \%$ y $54 \%$ de los indicadores respectivos, siendo posible el mejoramiento.

Competente: si el docente cumple con el $85 \%$ y $70 \%$ de los indicadores correspondientes, siendo deseable el mejoramiento

Destacado: si el docente cumple con el $85 \%$ a $100 \%$ de los indicadores correspondientes por tanto excede los requerimientos.

\section{Grados}

A. Tiene dificultad en participar y colaborar con compañeros. Tiene dificultad para aportar y recibir ideas, incapacidad de entender la diversidad de pensamiento o de acción de los miembros del equipo.

B. En ocasiones participa en las decisiones y proyectos que se plantean, colabora de manera poco efectiva con sus compañeros. Actitud poco proactiva dentro del equipo de trabajo

C. Participa y colabora, aporta conocimientos al grupo de trabajo. Actitud proactiva para el funcionamiento del grupo de trabajo. 
D. Se integra proactivamente en sesiones de trabajo colectivo, participando activamente en proyectos y actividades de su área. Alta capacidad de entregar los conocimientos e ideas propias a un grupo para lograr un cometido de manera colectiva, busca aportar.

Si se evalúa por tablas descriptivas, y el intervalo entre ellas es del $15 \%$, entonces una brecha pequeña es de $15 \%$ (un nivel por debajo), una moderada puede ser de $30 \%$ (dos niveles por debajo) y mayor sería $45 \%$ (tres niveles por debajo). Estas variaciones acontecen "a saltos". No obstante, esto puede corregirse al incorporar las nociones de valor central y tendencias, o sea, la persona puede resultar evaluada así: a. responde al conjunto de conductas observables del perfil; b responde parcialmente, tendiendo al perfil inmediato anterior, o corresponde al perfil, tendiendo a conductas propias del nivel inmediato superior.

La evaluación por indicadores permite mayor precisión en las brechas, ya que los valores resultantes pueden extenderse de manera armónica, sin saltos entre los rangos. En esta vía metodológica, una brecha pequeña sería inferior o igual al $15 \%$, una moderada sería superior al 15 y hasta un $20 \%$, una considerable sería entre un 21 y un $30 \%$, y crítica sería superior al $40 \%$, entendiendo que nunca se sobrepasa del $45 \%$, porque es el límite inferior del espectro.

\section{CONCLUSIONES.}

Realizando una evaluación general y resumida de la investigación, se considera que se ha contribuido con la Universidad
Francisco de paula Santander Ocaña, en la medida que se presenta una mirada vasta y honda referente a distintas visiones teóricas de gran relevancia, sobre el tema de la competencia docente.

En segundo lugar, se hace una exposición de los distintos modelos sobre competencia docente, lo cual es una prueba seria para llevar y revelar el camino a los futuros trabajos que pretendan explicar la competencia docente.

Por otro lado, el estudio realizado da lugar a pensar seriamente sobre la labor docente, la cual debe partir de la reflexión continua y permanente del quehacer pedagógico, de tal manera que esa misma reflexión le permita desarrollar las competencias necesarias para que su labor docente se vea reflejada en el producto final: los profesionales que forma.

El profesorado en el auto-análisis y el auto dramatización de algunos incidentes críticos acaecidos durante el ejercicio profesional, pueden ser una excelente vía para un cambio consistente de las competencias profesionales docentes

\section{FINANCIACIÓN}

Esta investigación cuenta con el apoyo institucional de la Universidad Francisco de Paula Santander Ocaña.

\section{BIBLIOGRAFÍA}

Barraza, A. (2007). La Formación Docente Bajo Una Conceptualización Comprehensiva y Un Enfoque Por Competencias XXXIII $\mathrm{N}^{\circ}$ 2. Revista Estudios Pedagógicos. 
Bezanilla, M. (julio de 2003). El Proyecto Tuning y las Competencias Específicas. Orientaciones pedagógicas para la convergencia europea de Educación Superior. Europa: Universidad de Deusto.

Bogoya, D., \& Torrado, M. (2000). Educar Para el Desarrollo de las Competencias - Una Propuesta para Reflexionar. En Competencias y Proyectos Pedagógico. Bogotá: Universidad Nacional de Colombia.

Bozu, Z., \& Canto, P. (2009). El Profesorado Universitario en la Sociedad del Conocimiento. Competencias Profesionales Docente. Revista de Formación e Innovación Educativa Universitaria, 87-97.

Cano García, M. (2008). La Evaluación Por Competencia en Educación Superior. Profesorado Revista de Curriculum y Formación de Profesorado. Recuperado Julio 9 de 2015. Http: //www.ugr.es/ recfpro/rev123COL1.pdf, 78.

Castro, A. (2007). Formación Inicial y Profesión Docente. Santiago: Universidad Diego Portales.

Chiavenato, I. (2001). Administración de recursos Humanos. Bogotá. $5^{\circ}$ dición: Mc Graw Hill.

Cruz, M., \& Martínez, M. (2012). Perfeccionamiento de un instrumento para la selección de expertos en las investigaciones educativas. Revista Electrónica de Investigación Educativa, 14(2).

Díaz Barriga, F. (2006). Enseñanza Situada: Vínculo entre la Escuela y la Vida. México: McGrawHill.

Domínguez, G. (2004). La sociedad del conocimiento, la formación por competencias y el nuevo rol de la
Universidad: nuevas necesidades de reestructuración y Configuración de los Planes de Estudio. Ponencia presentada en el XVIII Congreso chileno de Educación en Ingeniería: La Formación en Ingeniería basada en Competencias. Chile: Universidad del Bío-Bío, Concepción, Chile.

Echeverría, B. (2002). Gestión de la Competencia de Acción Profesional. Revista de Investigación Educativa. 1 Volumen 20, 7-43.

García Cabrero, B., Loredo, J., Luna, E., \& Rueda, M. (2008). Modelo de. Revista Iberoamericana de evaluación Educativa Volumen 1 Numero 3 consultado http://www.rinace.net/riee/numeros/vol1nu m3_e/art8.pdf.

Gonzalez, J., \& Wagenaar, R. (2003). : Tuning Educational Structures in Europe: Informe. Disponible en la web: http://www.relint.deusto.es/TUNINGProject /spanish/doc_fase1/Tuning\%20Education: (consulta Febrero 18 de 2015).

Guzmán Ibarra, I., \& Marín Uribe, R. (2011). La Competencia y las Competencias Docentes: Reflexiones sobre el concepto y la Evaluación. Revista Electrónica Interuniversitaria de Formación del Profesorado, 151-172.

Guzmán Ibarra, I., \& Marín Uribe, R. (7 de abril de 2015). Aufop. Obtenido de http://www.aufop.com/aufop/uploaded_files /articulos/1301588498.pdf

Nieto, L., \& Díaz, M. (2009). Diseño Curricular y Competencia Profesional. "Hacia la Evaluación de la Calidad en la Educación Agrícola Superior en México". México: COMEAA.

Pavié Nova, A. (Septiembre de 2012). Las Competencias Profesionales Del Profesorado de Lengua Castellana y 
Comunicaciones en Chile: Aportaciones a la Formación Inicial. Tesis Doctoral. Chile, Valladolid: Universidad de Valladolid, Facultad educación y Trabajo Social, Departamento Didáctica de la Lengua y Literatura.

Pavié, A. (2011). Formación docente: hacia una definición del concepto de competencia profesional docente. Revista Electrónica Interuniversitaria de Formación del Profesorado, 14(1), 67-80.

Pérez Gómez, A. (2007). Profesorado y Otros Profesionales de la Educación. Barcelona: Octaedro.

Rueda Beltran, M. (2009). La Evaluación del Desempeño Docente: Consideraciones Desde El Enfoque Por Competencias. Revista Electrónica de Educación, 11(2), 1-16.

Ruiz Iglesias, M. (2005). La Formación de Competencias en Educación: Una Mirada Detenida en las Competencias de la Profesión Docente. España:

Sarramorra, J. (2005). Teorías e Instituciones Contemporáneas de la Educación. 4 Edición. Barcelona: Ariel.

Spencer, L., \& Spencer, S. (2003). Competencias en el Trabajo. Modelo Para Un Rendimiento Superior. . Gestión Turística Numero VII Link http://www.diba.es/fl/fitxers/pfm057esp.PDF.

Tejada, J., \& Navio, A. (2005). “El desarrollo y la gestión de competencias profesionales: una mirada desde la formación". Revista Iberoamericana de Educación Número 37.

Tobón, S. (2007). El Enfoque Complejo de Las Competencias y el Diseño
Curricular Por Ciclos Propedéuticos. Acción Pedagógica, 14-28.

Valladares, L. (2010). Las Competencias en la Educación Científica: Tensiones desde el Pragmatismo Epistemológico. Perfiles Educativos Volumen XXXIII, Número 132, 158-179.

Zabala, A., \& Laia, A. (2007). Once Ideas Clave: Cómo Aprender y Enseñar Competencias. México: Colofón.

Zabalza, M. (2002). La Enseñanza Universitaria. Madrid: Narcea.

Zabalza, M. (2003). Competencias Docente del Profesional Universitario: Calidad y Desarrollo Profesional. Barcelona: Narcea.

Zabalza, M. (2009). Competencias Docente del Profesional Universitario. Calidad y Desarrollo Profesional. Madrid: Narcea. 